\title{
Sensor Fault Masking of a Ship Propulsion System
}

\author{
Wu, N. Eva; Thavamani, Shuda; Zhang, Youmin; Blanke, Mogens
}

Published in:

Control Engineering Practice

Link to article, DOI:

10.1016/j.conengprac.2005.09.003

Publication date:

2005

Link back to DTU Orbit

Citation (APA):

Wu, N. E., Thavamani, S., Zhang, Y., \& Blanke, M. (2005). Sensor Fault Masking of a Ship Propulsion System. Control Engineering Practice, 14(11), 1337-1345. https://doi.org/10.1016/j.conengprac.2005.09.003

\section{General rights}

Copyright and moral rights for the publications made accessible in the public portal are retained by the authors and/or other copyright owners and it is a condition of accessing publications that users recognise and abide by the legal requirements associated with these rights.

- Users may download and print one copy of any publication from the public portal for the purpose of private study or research.

- You may not further distribute the material or use it for any profit-making activity or commercial gain

- You may freely distribute the URL identifying the publication in the public portal

If you believe that this document breaches copyright please contact us providing details, and we will remove access to the work immediately and investigate your claim 


\title{
Sensor fault masking of a ship propulsion system
}

\author{
N. Eva $\mathrm{Wu}^{\mathrm{a}, *}$, Sudha Thavamani ${ }^{\mathrm{a}}$, Youmin Zhang ${ }^{\mathrm{b}}$, Mogens Blanke ${ }^{\mathrm{c}}$ \\ ${ }^{a}$ Department of Electrical and Computer Engineering, Binghamton University, Binghamton, NY 13902, USA \\ ${ }^{\mathrm{b}}$ Department of Computer Science and Engineering, Aalborg University Esbjerg, DK-6700, Esbjerg, Denmark \\ ${ }^{\mathrm{c}}$ Oersted.DTU, Technical University of Denmark, DK-2800 Kgs. Lyngby, Denmark
}

Received 30 December 2004; accepted 16 September 2005

Available online 11 November 2005

\begin{abstract}
This paper presents the results of a study on fault-tolerant control of a ship propulsion benchmark [Izadi-Zamanabadi, R., \& Blanke, M. (1999). A ship propulsion system as a benchmark for fault tolerant control. Control Engineering Practice, 7 (2), 227-239] which uses estimated or virtual measurements as feedback variables. The estimator operates on a self-adjustable design model so that its outputs can be made immune to the effects of a specific set of component and sensor faults. The adequacy of sensor redundancy is measured using the control reconfigurability [Wu, N. E., Zhou, K., \& Salomon, G. (2000). Reconfigurability in linear time-invariant systems. Automatica, 36 (11), 1767-1771] and the number of sensor based measurements are increased when this level is found inadequate. As a result, sensor faults that are captured in the estimator's design model can be tolerated without the need for any reconfiguration actions. Simulations for the ship propulsion benchmark show that, with additional sensors added as described and the estimator in the loop, satisfactory faulttolerance is achieved under two additive sensor faults, an incipient fault, and a parametric fault, without having to alter the original controller in the benchmark.
\end{abstract}

(C) 2005 Elsevier Ltd. All rights reserved.

Keywords: Reconfigurability; Sensor redundancy; Fault tolerant control; Adaptive estimation; Ship propulsion benchmark

\section{Introduction}

Sensor fault tolerant control for marine/aerospace vehicles is less discussed in the literature than actuator or component fault tolerant control, despite the large number of publications in the field following the overview paper by Patton (1997). The reasons may be that sensor reliability is high and hardware (direct) sensor redundancy is often used where measurements are critical. There are applications, however, where sensor faults are critical for the overall system. This is the case in the ship propulsion benchmark (Izadi-Zamanabadi \& Blanke, 1999). The aim of the benchmark was to demonstrate enhanced propulsion plant availability by autonomous handling of various faults in machinery or sensors.

In the propulsion system, critical sensors are made redundant. Even if adequate sensor redundancy exists, it

\footnotetext{
*Corresponding author. Tel.: + 16077774375 ; fax: + 16077774464

E-mail addresses: evawu@binghamton.edu (N.E.Wu), ymzhang@cs.aaue.dk (Y.M. Zhang),mb@oersted.dtu.dk (M. Blanke).
}

needs to be properly managed to mask single point failures. If a critical feedback variable is measured by three or more identical sensors, a simple scheme of majority voting can mask a single sensor fault and provide the correct measurement of the variable. If none or only one extra sensor is associated with the variable, analytic redundancy (Chow \& Willsky, 1984) provided via the use of static or dynamic models that relate the system variables must be invoked in order to mask a sensor fault. An alternative approach is for the control law to react to a diagnosis outcome rather than to mask the effect of a fault. This more flexible approach to the management of redundancy is called control reconfiguration (Blanke, Kinnaert, Lunze, \& Staroswiecki, 2003). However, its implementation is more complex and may carry a higher decision risk $(\mathrm{Wu}$, 2004).

Two basic fault-tolerant approaches were listed by IzadiZamanabadi and Blanke (1999) in describing the ship propulsion benchmark problem. Accommodation by recalculation of the set points at the coordination level was suggested for some faults, reconfiguration by replacing a 
faulty measurement with an alternative output estimate was suggested for other faults. The latter approach was demonstrated in Blanke, Izadi-Zamanabadi, and Lootsma (1998), which depends, as in all reconfiguration approaches, on the timely isolation of faults. The set points recalculation can take effect only if adequate redundancy exists and the redundancy is properly managed. Though most passenger vessels have two complete shafts that can provide fail-operational propulsion systems, they lack the capability of effectively mitigating the impact of sensor faults that affect availability of full engine power and thus affect their regularity of service. For example, inclusion of an additional (redundant) shaft speed sensor is in accordance with the practice in the marine industry where the higher of the sensor signals is chosen in a maximum selector for use in the engine speed control loop. However, certain sensor faults could easily cause an unwanted engine slow down when this simple form of sensor management is used. Automatic deselection of the faulty signal following isolation of the faulty sensor or alternatively, masking of the faulty signal, would clearly be superior in enhancing propulsion plant availability.

Other approaches to solve the benchmark problem have employed several existing and new methods from diagnosis and control for linear and non-linear systems. Blanke et al. (1998) focused on the engine related faults in shaft speed sensor and engine gain. They designed an adaptive, nonlinear observer for fault estimation. When a fault was isolated, the faulty input was disabled from the control algorithm. The observer output was used as an estimate of shaft speed when a shaft speed sensor fault was diagnosed. A simple switching to this estimate from the faulty measurement managed controller reconfiguration. Research results from several groups and several conference papers (Amann, Perronne, Gissinger, \& Frank (1999), Blanke \& Lootsma (1999), Schreier \& Frank (1999), Kerrigan \& Maciejowski, and Edwards \& Spurgeon (2000)) were summarized in a book chapter (IzadiZamanabadi et al., 2000). Methods included structural analysis to provide residuals, linear methods employing observers and parity relations for diagnosis, model reference predictive control to accommodate faults, quantized modelling and diagnosis for the problem, and active controller reconfiguration using state-event logic in a supervisor. An adaptive two-stage extended Kalman filter based method was developed for fault diagnosis of the benchmark in Zhang and Wu (1999). Edwards and Spurgeon (2000) used a sliding mode observer for fault detection on the non-linear propulsion plant. Two Ph.D. theses used the benchmark as a main example. Supervisor logic design for the benchmark was a main theme in IzadiZamanabadi (1999). The nonlinear problem was the focus in Lootsma (2001). He used nonlinear geometric theory to formulate the residual generators for the complete problem and demonstrated detection and isolation for all faults considered in the benchmark. Bonivento, Paoli, and Marconi (2003) used a high gain observer for the non- linear shaft speed dynamics, similar to that of Blanke et al. (1998), however not adaptive, and they applied a static detector to diagnose pitch and engine gain faults. An estimate of the magnitude of sensor faults was used by the control scheme to accommodate faults by setpoint alteration. Reflecting on the nonlinear dynamics, uncertain parameters and complex designs with many earlier methods, Izadi-Zamanabadi, Blanke, and Katebi (2003) suggested a neuro-fuzzy output observer for diagnosis and showed this could cope with both the inaccuracy and nonlinear behavior of the system as well as partial lack of knowledge about certain parameters. A time-varying fuzzy threshold was used for detection. The benchmark was furthermore used in the textbook by Blanke et al. (2003) to illustrate the details of a complete fault-tolerant design.

The aim of this paper is to investigate techniques that make the propulsion system unaffected by faults in sensor systems without reconfiguration. The approach is to use an estimator to mask sensor faults through replacement of feedback variables by their estimates that are immune to sensor faults. Fault immunity is achieved by assuring a sensor arrangement that preserves system reconfigurability (Wu, Zhou, \& Salomon, 2000) before and after faults occur, and by capturing the effect of faults in the model used for the design of an estimator. It is shown possible to achieve fault-tolerance without having to alter control architecture. This approach is significantly different from reported approaches to fault-tolerant control of the ship propulsion benchmark and it has potential to solve other fault-tolerant control problems.

The paper is organized as follows. Section 2 discusses the basic concept of the proposed sensor fault masking approach. Section 3 describes the ship propulsion benchmark problem to which the proposed scheme is applied. It also presents the algorithm that provides the virtual measurements in the form of a set of recursive equations. Section 4 shows simulation results with a fault masking estimator in the control loop. Section 5 comments on the need for further research. Section 6 acknowledges external support for this work.

\section{Problem formulation}

Consider the tracking problem for a system with the following model:

$$
\begin{aligned}
\dot{x} & =f(x, \xi, u)+w_{x}, \quad \xi=\left[\begin{array}{ll}
\xi_{c}^{\mathrm{T}} & \xi_{s}^{\mathrm{T}}
\end{array}\right]^{\mathrm{T}}, \\
\dot{\xi} & =\alpha(x, \xi)+w_{\xi}, \\
y & =h\left(x, \xi_{s}\right)+w_{y}
\end{aligned}
$$

with

$y_{i}=x_{j_{i}}+\xi_{s, i}+w_{y, i}, \quad j_{i}=1, \ldots, q \leqslant n$.

The notations in the above equations are now explained. $x \in R^{n_{x}}$ is the state vector. $u \in R^{m}$ is the control input vector. $y \in R^{p}$ is the measured output vector. $w_{x} \in R^{n_{x}}$, $w_{\xi} \in R^{n_{\xi}}$, and $w_{y} \in R^{p}$ are bounded exogenous input 
vectors. $\xi$ is the fault effect parameter vector with $\xi_{c} \in$ $\Omega_{c} \subset R^{n_{c}}$ representing component faults, $\xi_{s} \in \Omega_{s} \subset R^{n_{s}}$ representing sensor faults, and $\xi=0$ representing the fault-free case. Let $x_{m}=\left[x_{j_{1}} \cdots x_{j_{q}}\right]^{\mathrm{T}}$ with $q$ the number of state variables which are measured and participate feedback. Note that the form of the measurement equation is specialized to the ship propulsion benchmark. The upper block diagram in Fig. 1 shows the configuration with the following conventional control law:

$\dot{x}_{c}=g\left(x_{c}, y, r\right), \quad u=\gamma\left(x_{c}, y, r\right)$

which is designed for $\xi=0$ to achieve

$\left\|x_{m}(t)-r(t)\right\| \leqslant \varepsilon, \quad \forall t \geqslant T$,

where $\varepsilon>0$ is a pre-specified tracking error bound, $T>0$ is a pre-specified settling time bound, and $r \in R^{q}$ is the reference input vector. In general, it is not possible to find a control law by which (6) is satisfied $\forall \xi_{c} \in \Omega_{c}$ and $\forall \xi_{s} \in \Omega_{s}$ because the feedback variable $y$ is affected by sensor and component faults. The following fault-tolerant control law is proposed, as shown in the lower schematic diagram of Fig. 1. The new component in the control law is an estimator expressed as

$\dot{\hat{x}}=\phi(\hat{x}, \hat{\xi}, u, y, r)$,

$\dot{\hat{\xi}}=\kappa(\hat{x}, \hat{\xi}, u, y, r)$,

$\hat{x}_{m}=h\left(\hat{x}, \hat{\xi}_{s}\right)$,

where $\hat{x}$ is the state estimate vector, $\hat{\xi}$ is the fault-effect parameter estimate vector, and $\hat{x}_{m}$ is the virtual measurement vector. The estimator is followed by the original controller with the sensor measurement $y$ replaced by the virtual measurement $\hat{x}_{m}$

$\dot{x}_{c}=g\left(x_{c}, \hat{x}_{m}, r\right), \quad u=\gamma\left(x_{c}, \hat{x}_{m}, r\right)$.

If $\hat{x}_{m}$ remains close to $x_{m}=h\left(x, \xi_{s}\right)$ for all $\xi_{s} \in \Omega_{s}$, sensor faults are said to have been masked. In this case, the estimator is said to have acquired self-adjustability with respect to the occurrence of sensor faults. If, in addition, the tracking condition $\left\|x_{m}(t)-r(t)\right\| \leqslant \varepsilon \forall t \geqslant T$ is satisfied as well for all $\xi_{s} \in \Omega_{s}$ and $\xi_{c} \in \Omega_{c}$, the controlled system is said to be sensor fault-tolerant, and the controller described in (8) is said to be robust against component faults. Keeping a part of observer-based controller (8) in the same form as conventional controller (5) is well justified under the conditions that conventional controller (5) performs satisfactorily in the nominal situation, that minimization of control hardware/software change is always desirable, and that the addition of a decision module that triggers control reconfiguration is avoided.

Two issues must be addressed. The first is the characterization of a model's structural property that is necessary in order to mask sensor faults. The second is the construction of the estimator that achieves fault masking. The general principles to resolving these issues are briefly discussed now. How they are dealt with in the ship propulsion benchmark will be detailed in the next section.

The structural property sought after is the control reconfigurability ( $\mathrm{Wu}$, Zhou, et al., 2000) that measures the adequacy of redundancy level in a controlled system relative to the nominal condition. Numerically it is a combined measure of controllability and observability under specified fault scenarios. Explicit formulae for the computation of a control reconfigurability are available, however, only for small signal (linear) models. Nevertheless the computation is straightforward in this case, and the results are instructive with regard to the selection of alternative sensor and actuator arrangement. In order for the same conventional controller (5) to be effective before and after some sensor faults have occurred, the control reconfigurability specific to these sensor faults should necessarily remain approximately unchanged. This condition, however, is not sufficient unless the sensor faults are masked via redundancy management and the conventional controller is robust against the remaining faults.

The sensor fault masking can be realized using an estimator (Wu, Zhang, \& Zhou, 2000), and in particular using an extended Kalman estimator for the ship propulsion system (Zhang \& Wu, 1999). The role of parameter estimates pertinent to the application of the ship propulsion benchmark can be viewed as a means to adjust the estimator design model according to the values of the fault effect parameters so that the virtual measurement quantities are always correct $\left(\hat{x}_{m} \approx x_{m}\right)$. An important feature of the estimator is the use of a set of temporally and spatially varying forgetting factors that facilitate the estimator

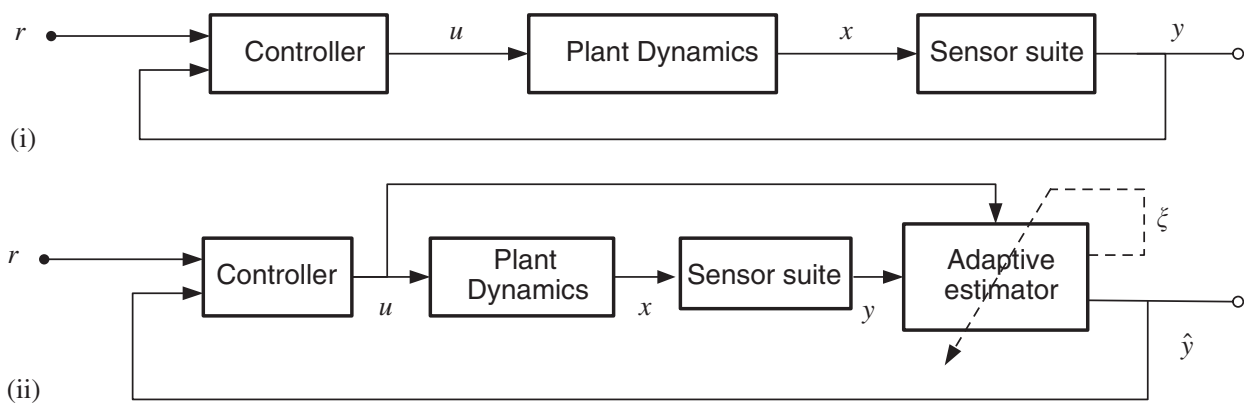

Fig. 1. (i) Original system configuration, (ii) Fault-tolerant system configuration 
convergence and minimize the dynamic effects of the estimator to the control loop.

\section{Ship propulsion control system}

The ship propulsion benchmark is a test bed for fault detection, diagnosis and fault-tolerant control. The lower level subsystems include a propeller pitch angle control subsystem, a governor subsystem, a diesel engine, a propeller, and a ship speed subsystem. The reader is referred to Izadi-Zamanabadi and Blanke (1999) for a detailed description. The model described here is the design model for the fault masking estimator in which all faults entering the system are manipulated into additive random fault effect parameters. The set of fault effect parameters includes two additive sensor faults $(\Delta \theta, \Delta n)$, an additive incipient fault $\left(\Delta \dot{\theta}_{i n c}\right)$, and a multiplicative parametric fault $\left(\Delta k_{y}\right)$.

The model based on which the estimator design is carried out has the state-space representation given in (1), (2), and (3)

$$
\begin{aligned}
& \dot{x}=f(x, \xi, u)+w_{x}, \quad \dot{\xi}=\alpha(x, \xi)+w_{\xi}, \\
& y=h\left(x, \xi_{s}\right)+w_{y},
\end{aligned}
$$

where

$$
\begin{aligned}
& x=\left[\begin{array}{c}
\theta \\
n \\
U \\
Q_{\text {eng }}
\end{array}\right]=\left[\begin{array}{c}
\text { propeller pitch } \\
\text { shaft speed } \\
\text { ship speed } \\
\text { engine torque }
\end{array}\right], \\
& x_{m}=\left[\begin{array}{c}
\theta \\
n \\
U
\end{array}\right], \\
& \xi=\left[\begin{array}{c}
\xi_{s} \\
\xi_{c}
\end{array}\right]=\left[\begin{array}{c}
\Delta \theta \\
\Delta n \\
\Delta \dot{\theta}_{i n c} \\
\Delta k_{y}
\end{array}\right] \\
& =\left[\begin{array}{c}
\text { pitch sensor fault } \\
\text { shaft speed sensor fault } \\
\text { pitch rate drift } \\
\% \text { engine gain reduction }
\end{array}\right] \text {, } \\
& u=\left[\begin{array}{c}
\theta_{r e f} \\
Y_{m}
\end{array}\right]=\left[\begin{array}{l}
\text { pitch angle reference } \\
\text { measured fuel index }
\end{array}\right], \\
& y=\left[\begin{array}{c}
\theta_{m} \\
n_{m} \\
U_{m}
\end{array}\right]=\left[\begin{array}{c}
\text { measured propeller pitch } \\
\text { measured shaft speed } \\
\text { measured ship speed }
\end{array}\right]
\end{aligned}
$$

and the bounded exogenous signals

$$
\begin{aligned}
& w_{x}=\left[\begin{array}{c}
v_{\theta} \\
0 \\
0 \\
0
\end{array}\right], \quad w_{\xi}=\left[\begin{array}{c}
v_{\Delta \theta} \\
v_{\Delta n} \\
v_{\Delta \dot{\theta}_{i n c}} \\
v_{\Delta k_{y}}
\end{array}\right], \\
& w_{y}=\left[\begin{array}{c}
v_{\theta_{m}} \\
v_{n_{m}} \\
v_{U_{m}}
\end{array}\right] .
\end{aligned}
$$

Functions $f, \phi$ and $h$ are given by

$$
\begin{aligned}
& f(x, \xi, u) \\
& =\left[\begin{array}{l}
\max \left(\dot{\theta}_{\text {min }}, \min \left\{k_{t}\left[\theta_{\text {ref }}-(\theta+\Delta \theta)\right], \dot{\theta}_{\text {max }}\right\}\right)+\Delta \dot{\theta}_{\text {inc }} \\
\left(Q_{\text {eng }}-Q_{\text {prop }}\right) / I_{m} \\
\left(\left(1-t_{T}\right) T_{\text {prop }}-R_{U}\right) / m \\
\left(\left(1+\Delta k_{y}\right) k_{y} Y_{m}-Q_{\text {eng }}\right) / \tau_{c}
\end{array}\right], \\
& \phi(x, \xi)=\xi \text { and } h\left(x, \xi_{s}\right)=\left[\begin{array}{l}
\theta+\Delta \theta \\
n+\Delta n \\
U
\end{array}\right] .
\end{aligned}
$$

Additional variables in the functions are defined as follows. $\dot{\theta}_{\min }$ and $\dot{\theta}_{\max }$ are the pitch rate limits. $I_{m}$ and $m$ are the inertia and the mass of the ship, respectively. $R_{U}$ is the hull resistance. $k_{y}$ is the diesel engine gain and $\tau_{c}$ is the engine time constant corresponding to torque built up from cylinder firings. $Q_{\text {prop }}$ and $T_{\text {prop }}$ are developed propeller torque and thrust, respectively. In the benchmark simulation, they are calculated by the interpolation of two tables of real data measured under sea operation. They are nonlinear functions of $\theta, n$, and ship speed $U$, approximated by the following bilinear relations:

$Q_{\text {prop }}=Q_{0}|n| n+Q_{|n| n}(\theta)|n| n+Q_{|n| V_{a}}(\theta)|n| V_{a}$,

$T_{\text {prop }}=T_{|n| n}(\theta)|n| n+T_{|n| V_{a}}(\theta)|n| V_{a}$,

where $T_{|n| n}, T_{|n| V_{a}}, Q_{|n| n}$ and $Q_{|n| V_{a}}$ are complex functions of $\theta, V_{a}$ is the advance speed. The relation between $V_{a}$ and $U$ can be described by the wake fraction $w$ through $V_{a}=(1-w) U$.

The decision concerning the addition of sensors is made based on a control reconfigurability calculation $(\mathrm{Wu}$, Zhou, et al., 2000), and an observability calculation (Moore, 1981).

With the added sensors, mapping $h\left(x, \xi_{s}\right)$ in the measurement Eq. (4), or more concretely in (9) that is used in the estimator design model, should be modified to

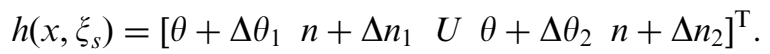

Despite the increase in the number of measurements, the states that participate the feedback remains to be the same components of $x_{m}$. 
Control reconfigurability assesses the system ability to allow performance restoration in the presence of faults. A control reconfigurability calculation is performed for the small signal control design model with $\dot{\theta}, Y_{m}$ as inputs, $\theta, n$, $U, Q$ as states. Four sets of outputs are considered, corresponding to the four options of sensor arrangement shown in the last column of Table 1: no added sensors (1p1s), an added pitch sensor (2p1s), an added shaft speed sensor (1p2s), and an added pitch sensor and an added shaft speed sensor (2p2s). Relative control reconfigurability, denoted by $\rho_{r}$, with respect to the nominal control reconfigurability for each sensor arrangement, is calculated under two sensor fault scenarios. The calculation involves finding the smallest relative Hankel singular value for a specific fault scenario. The two sensor fault scenarios are a single pitch sensor fault $(\Delta \theta)$, and a single shaft speed sensor fault $(\Delta n)$. To numerically calculate $\rho_{r}(\mathrm{Wu}$, Zhou, et al., 2000), let $X_{i \mathrm{pjs}}$ and $Y_{i \mathrm{ipjs}}$ be respectively stabilizing solutions to

$X_{i \mathrm{pjs}} A+A^{\mathrm{T}} X_{i \mathrm{pjs}}-X_{i \mathrm{pjs}} B B^{\mathrm{T}} X_{i \mathrm{p} j \mathrm{~s}}=0$

and

$A Y_{i \mathrm{pjs}}+Y_{i \mathrm{pjs}} A^{\mathrm{T}}-Y_{i \mathrm{pjs}} C_{i \mathrm{pjs}}^{\mathrm{T}} C_{i \mathrm{pjs}} Y_{i \mathrm{pjs}}=0$,

where $i, j=1,2,\left(A, B, C_{i \mathrm{pjs}}\right)$ is a minimum realization of the small signal model of ship propulsion system. Then solve for $P_{i \mathrm{pjs}}$ and $Q_{i \mathrm{pjs}}$ for each case from

$\left(A-B B^{\mathrm{T}} X_{i \mathrm{pjs}}\right) P_{i \mathrm{pjs}}+P_{i \mathrm{pjs}}\left(A-B B^{\mathrm{T}} X_{i \mathrm{pjs}}\right)^{\mathrm{T}}+B B^{\mathrm{T}}=0$

and

$$
\begin{aligned}
& Q_{i \mathrm{pjs}}\left(A-Y_{i \mathrm{pjs}} C_{i \mathrm{pjs}}^{\mathrm{T}} C_{i \mathrm{pjs}}\right)+\left(A-Y_{i \mathrm{pjs}} C_{i \mathrm{pjs}}^{\mathrm{T}} C_{i \mathrm{pjs}}\right)^{\mathrm{T}} Q_{i \mathrm{pjs}} \\
& \quad+C_{i \mathrm{pjs}}^{\mathrm{T}} C_{i \mathrm{p} j \mathrm{~s}}=0 .
\end{aligned}
$$

Control reconfigurability $\rho$ is the square root of the smallest eigenvalue of $P Q$, and the relative control reconfigurability is given by

$\rho_{r, i \mathrm{pj} s}=\left[\rho_{i \mathrm{p} j \mathrm{~s}}\right.$ at worst case $] /\left[\rho_{i \mathrm{p} j \mathrm{~s}}\right.$ in the nominal case $]$.
The above calculation is carried out for each of the four sensing arrangements with respect to a pitch sensor fault, and a shaft speed sensor fault, respectively. The results of the calculation are shown in the first components of the eight pairs in Table 2.

On the other hand, the ability to estimate the state of a dynamic system rests on the system's observability. Since all types of faults to be estimated must be captured in the estimator design model as states, an observability calculation is performed for the state augmented estimator design model with the same four options of sensor arrangement, where the feedback variable in the engine loop is $\theta$. The relative observability, denoted by $\sigma_{r}$, is defined as the ratio of the smallest singular value of an observability gramian to that of the $2 \mathrm{p} 2 \mathrm{~s}$ case. Two simplifications are made in the gramian calculations. One is the exclusion of faulteffect parameters $\Delta \dot{\theta}_{i n c}$ and $\Delta k_{y}$ from the calculation because they are not sensor related faults. The other is the perturbation of the open-loop poles at the origin associated with $\dot{\xi}_{s}=0$ to the open left half plane. The results of the relative observability computation are shown in the second components of the eight pairs in Table 2.

It is found that the state augmented models are observable under all sensor arrangements, though the observabilities are generally weak. The relative observability with 2 pitch angle sensors is markedly stronger than that with a single pitch sensor, as indicated in Table 2. It can also be seen from Table 2 that a single pitch sensor fault does not alter the system reconfigurability, while a single shaft speed fault significantly reduces the reconfigurability, unless a second shaft speed sensor is present. We therefore conclude that the only viable option that allows a successful sensor fault masking control is $2 \mathrm{p} 2 \mathrm{~s}$. The above conclusions are supported by our simulations. The simulation results shown in the paper are obtained with the $2 \mathrm{p} 2 \mathrm{~s}$ option.

With two pitch angle sensors and two shaft speed sensors, the reconfigurability is retained before and after

Table 1

Estimator design models under four sensor arrangements

\begin{tabular}{lllll}
\hline & Inputs & States & Fault-effect parameters & Outputs \\
\hline $1 \mathrm{p} 1 \mathrm{~s}$ & $\theta_{\text {ref }}, Y_{m}$ & $\theta, n, U, Q$ & $\Delta \theta, \Delta n$ & $\theta_{m}, n_{m}, U_{m}$ \\
$2 \mathrm{p} 1 \mathrm{~s}$ & $\theta_{r e f}, Y_{m}$ & $\theta, n, U, Q$ & $\Delta \theta_{1}, \Delta n, \Delta \theta_{2}$ & $\theta_{m 1}, n_{m}, U_{m}, \theta_{m 2}$ \\
$1 \mathrm{p} 2 \mathrm{~s}$ & $\theta_{r e f}, Y_{m}$ & $\theta, n, U, Q$ & $\Delta \theta_{1}, \Delta n_{1}, \Delta n_{2}$ & $\theta_{m 1}, n_{m 1}, U_{m}, n_{m 2}$ \\
$2 \mathrm{p} 2 \mathrm{~s}$ & $\theta_{r e f}, Y_{m}$ & $\theta, n, U, Q$ & $\Delta \theta_{1}, \Delta n_{1}, \Delta \theta_{2}, \Delta n_{2}$ & $\theta_{m 1}, n_{m 1}, U_{m}, \theta_{m 2}, n_{m 2}$ \\
\hline
\end{tabular}

Table 2

Relative reconfigurability $\rho_{r}$ and relative observability $\sigma_{r}$ for four options of sensor arrangement

\begin{tabular}{lllll}
\hline$\left(\rho_{r}, \sigma_{r}\right)$ & $1 \mathrm{p} 1 \mathrm{~s}$ & $2 \mathrm{p} 1 \mathrm{~s}$ & $1 \mathrm{p} 2 \mathrm{~s}$ & $2 \mathrm{p} 2 \mathrm{~s}$ \\
\hline$\Delta \theta$ & $(1.000,0.000)$ & $(1.000,54.863)$ & $(1.000,0.000)$ & $(1.000,1.000)$ \\
$\Delta n$ & $(0.018,0.000)$ & $(0.018,54.863)$ & $(0.729,0.000)$ & $(0.729,1.000)$ \\
\hline
\end{tabular}




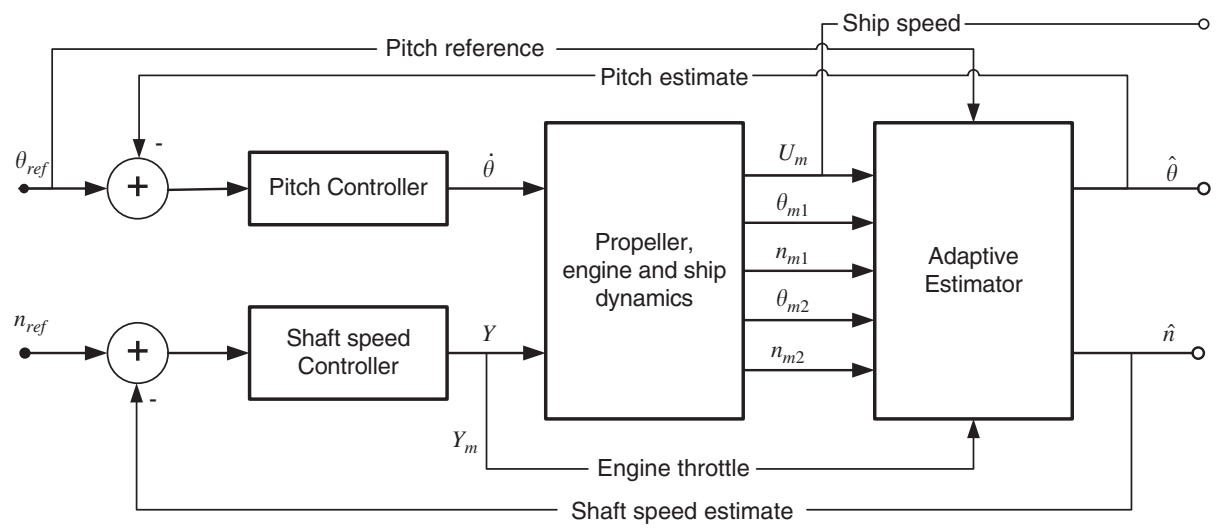

Fig. 2. A fault-tolerant control scheme with sensor fault-masking virtual measurements.

the occurrence of single sensor faults, and the observability necessary for the estimation of the propulsion system states and the sensor fault effect parameters is greatly enhanced. As a result, accurate estimates can be expected of the feedback variables $\theta$ and $n$. A fault masking control scheme has been devised with an estimator in the original controlloop. The estimator generates the virtual measurements that replace the feedback variables provided by the two original pitch angle and shaft speed sensors. The on-line estimator has been added to the Simulink model of the original benchmark (Thavamani, 2002). The sensor fault masking schematic is shown in Fig. 2.

\section{Estimation algorithm}

An estimator based on a 1 sample/second discretized version of the model given in Section 3 was designed for the purpose of fault detection and diagnosis in the $1 \mathrm{p} 1 \mathrm{~s}$ case (Zhang \& Wu, 1999). Two major modifications with regard to the estimator design have been introduced in the current paper. (i) The estimator design model now includes an additional pitch angle sensor, and an additional shaft speed sensor, as well as the additional fault effect parameters, as shown in (10), and (ii) the estimator is implemented as a Matlab routine which is placed in the control loop of the benchmark Simulink model.

\subsection{Bias-separated discrete time model with redundant} sensors

$$
\begin{aligned}
& x(k+1)=f(x(k))+G(k) u(k)+F_{1}(k) b(k) \\
& \quad+\Gamma(k) w^{x}(k), \\
& b(k+1)=b(k)+w^{b}(k), \\
& y(k+1) \\
& \quad=h(x(k+1))+F_{2}(k+1) b(k+1)+v(k+1),
\end{aligned}
$$

where, State vector:

$x=\left[\begin{array}{llll}\theta & n & U & Q_{\text {eng }}\end{array}\right]^{\mathrm{T}}$.
Control input vector:

$u=\left[\begin{array}{ll}\theta_{r e f} & Y_{m}\end{array}\right]^{\mathrm{T}}$.

Measurement vector:

$z=\left[\begin{array}{lllll}\theta_{m 1} & n_{m 1} & U_{m} & \theta_{m 2} & n_{m 2}\end{array}\right]^{\mathrm{T}}$.

Fault parameter vector:

$b=\left[\begin{array}{llllll}\Delta \theta_{1} & \Delta n_{1} & \Delta \dot{\theta}_{\text {inc }} & \Delta k_{y} & \Delta \theta_{2} & \Delta n_{2}\end{array}\right]^{\mathrm{T}}$.

System noise vector:

$w^{x}=\left[\begin{array}{llllll}v_{\theta 1} & 0 & 0 & 0 & v_{\theta 2} & 0\end{array}\right]^{\mathrm{T}}$.

Measurement noise vector:

$v=\left[\begin{array}{lllll}v_{\theta 1} & v_{n 1} & v_{U} & v_{\theta 2} & v_{n 2}\end{array}\right]^{\mathrm{T}}$

and

$$
\begin{aligned}
& f(x(k))=\left[\begin{array}{c}
\left(1-k_{t} T\right) \theta(k) \\
n(k)+\frac{T}{I_{m}} Q_{\text {eng }}[x(k), k] \\
-\frac{T}{I_{m}} Q_{\text {prop }}[x(k), k] \\
\left(1-t_{T}\right) T \\
m \\
U(k)+\frac{T}{m} R_{U}[x(k), k] \\
\left(1-\frac{T}{\tau_{C}}\right) Q_{\text {eng }}[x(k), k]
\end{array}\right], \\
& G(k)=\left[\begin{array}{cc}
k_{t} T & 0 \\
0 & 0 \\
0 & 0 \\
0 & \frac{T}{\tau_{c}} k_{y}
\end{array}\right], \\
& F_{1}(k)=\left[\begin{array}{cccccc}
-k_{t} T & 0 & T & 0 & -k_{t} T & 0 \\
0 & 0 & 0 & 0 & 0 & 0 \\
0 & 0 & 0 & 0 & 0 & 0 \\
0 & 0 & 0 & \frac{k_{y} T}{\tau_{C}} Y_{m}(k) & 0 & 0
\end{array}\right]
\end{aligned}
$$


$\Gamma(k)=\left[\begin{array}{cccccc}-k_{t} T & 0 & 0 & 0 & -k_{t} T & 0 \\ 0 & 0 & 0 & 0 & 0 & 0 \\ 0 & 0 & 0 & 0 & 0 & 0 \\ 0 & 0 & 0 & 0 & 0 & 0\end{array}\right]$

$h(x(k+1))=\left[\begin{array}{llll}1 & 0 & 0 & 0 \\ 0 & 1 & 0 & 0 \\ 0 & 0 & 1 & 0 \\ 1 & 0 & 0 & 0 \\ 0 & 1 & 0 & 0\end{array}\right]$,

$F_{2}(k)=\left[\begin{array}{cccccc}1 & 0 & 0 & 0 & 0 & 0 \\ 0 & 1 & 0 & 0 & 0 & 0 \\ 0 & 0 & 0 & 0 & 0 & 0 \\ 0 & 0 & 0 & 0 & 1 & 0 \\ 0 & 0 & 0 & 0 & 0 & 1\end{array}\right]$.

The noise sequences $w^{x}, w^{b}, v$ are assumed to be zero mean uncorrelated Gaussian noise sequences with

$$
\begin{gathered}
E\left\{\left[\begin{array}{c}
w^{x}(k) \\
w^{b}(k) \\
v(k)
\end{array}\right]\left[\begin{array}{lll}
w^{x}(j) & w^{b}(j) & v(j)
\end{array}\right]\right\} \\
=\left[\begin{array}{ccc}
Q^{x} & 0 & 0 \\
0 & Q^{b} & 0 \\
0 & 0 & R
\end{array}\right] \delta_{k j},
\end{gathered}
$$

where $Q^{x}>0, Q^{b}>0, R>0$ and $\delta_{k j}$ is the Kronecker delta.

The fault masking estimation algorithm involves a set of recursive equations, as shown in the following, which are used to produce estimates of both the states and the fault effect parameters. These are the same set of equations as those in Zhang and Wu (1999) with two exceptions as mentioned previously. That is: (i) modified measurement equations (10) replaces the original measurement equation, and (ii) the resulting estimator is implemented on-line using a Matlab function. Following a similar route of derivation as in Zhang and $\mathrm{Wu}$ (1999), the bias-separated extended Kalman filter for the above system are obtained. For the sake of brevity, the time index of the following equations has been dropped.

\subsection{An adaptive bias-separated extended Kalman Filter}

Bias-free state estimator:

$\tilde{x}^{+}=f+G u+\left(M-V^{+}\right) \hat{b}$,

$\tilde{P}^{x+}=F \tilde{P}^{x} F^{\prime}+\Gamma^{x} Q^{x} \Gamma^{\prime x}+M P^{b} M^{\prime}-V^{+} P^{b+} V^{\prime+}$,

$\tilde{x}=\tilde{x}^{+}+\tilde{K}^{x} \tilde{r}$

$\tilde{K}^{x}=\tilde{P}^{x} H^{\prime}[\tilde{S}]^{-1}$,
$\tilde{P}^{x}=\left(I_{x}-\tilde{K}^{x} H\right) \tilde{P}^{x+}$,

where the filter residual vector and its covariance are given as

$\tilde{r}=y-h\left(\tilde{x}^{+}\right)$,

$\tilde{S}=H \tilde{P}^{x} H^{\prime}+R$.

The Jacobians in the Taylor series expansion for non-linear functions $f$ and $h$ are

$F=\left.\frac{\partial f}{\partial x}\right|_{x=\tilde{x}}, \quad H=\left.\frac{\partial h}{\partial x}\right|_{x=\tilde{x}^{+}}$.

Bias estimator:

$P^{b+}=P^{b}+Q^{b}$,

$\hat{b}=\hat{b}+K^{b}(\tilde{r}-N \hat{b})$,

$K^{b}=P^{b+} N^{\prime}\left[N P^{b+} N^{\prime}+\tilde{S}\right]^{-1}$,

$P^{b}=\left(I_{b}-K^{b} N\right) P^{b+}$.

Coupling equations:

$M=F V+F_{1}$,

$V^{+}=M P^{b}\left[P^{b+}\right]^{-1}$,

$N=H V^{+}+F_{2}$,

$V=V^{+}-\tilde{K}^{x} N$.

Compensated state estimate:

$\hat{x}=\tilde{x}+V \hat{b}$.

Propeller pitch estimate:

$\hat{\theta}=\hat{x}(1)$.

Shaft speed estimate:

$\hat{n}=\hat{x}(2)$.

Estimate output:

Estimate $=\left[\begin{array}{ll}\hat{\theta} & \hat{n}\end{array}\right]$.

To make the above estimation algorithm more responsive to the changes of bias parameters which model the faults, forgetting factor technique has been introduced into the bias covariance (32)

$P^{b+}=\sum_{i=1}^{p} \frac{\alpha_{i}}{\lambda_{i}} e_{i} e_{i}^{\mathrm{T}}+Q^{b}, \quad 0<\lambda_{i} \leqslant 1$.

The forgetting factor $\lambda_{i}$ can be chosen as a decreasing function of the amount of information received in the direction $e_{i}$. Since eigenvalue $\alpha_{i}$ of $P^{b}$ is a measure of the uncertainty in the direction of $e_{i}$, a choice of forgetting factor $\lambda_{i}$ can be

$\lambda_{i}= \begin{cases}1, & \alpha_{i}>\alpha_{\max }, \\ \alpha_{i}\left[\alpha_{\min }+\frac{\alpha_{\max }-\alpha_{\min }}{\alpha_{\max }} \alpha_{i}\right]^{-1}, & \alpha_{i} \leqslant \alpha_{\max } .\end{cases}$

The reader is referred to Zhang and Wu (1999) for more discussion on the choice of forgetting factors. 


\section{Simulation results}

Figs. 3-5 show the responses of the measured variables $\theta_{m}, n_{m}$, and $U_{m}$ of the original benchmark simulation (first plot in each figure), the responses of $\theta_{m}, n_{m}$, and $U_{m}$ when the sensor fault masking control is implemented (last plot in each figure), and the responses of the true state $\theta, n$, and $U$ under the fault masking setting (middle plot in Figs. 2 and 3, last plot in Fig. 4 for $U=U_{m}$ in the benchmark). It
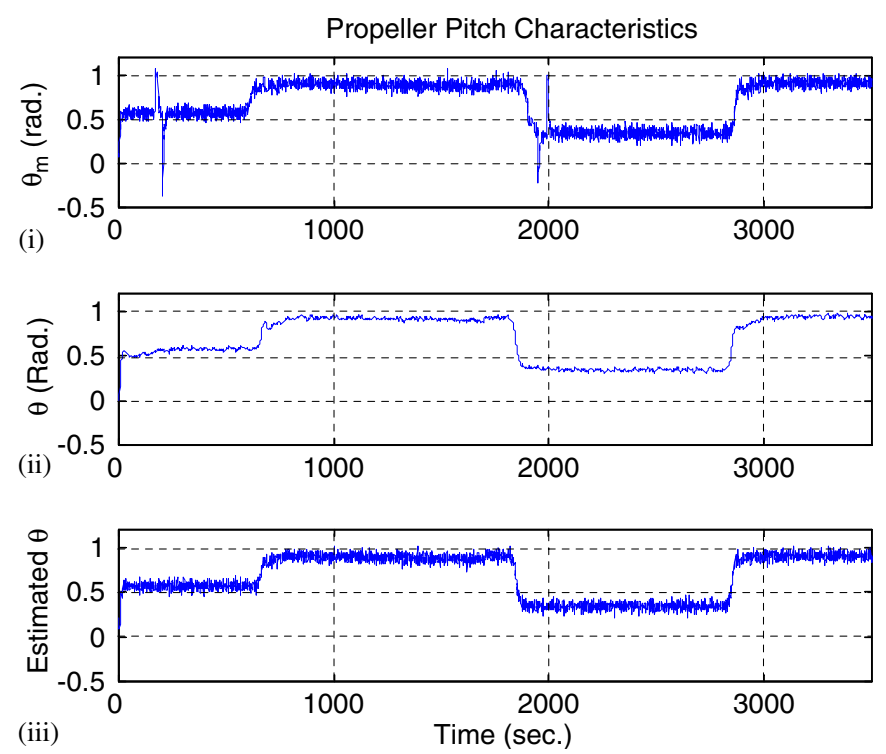

Fig. 3. Pitch angle (i) measurement from the original benchmark, (ii) true state from the fault-tolerant system, (iii) estimate that is fed back in the fault-tolerant system.
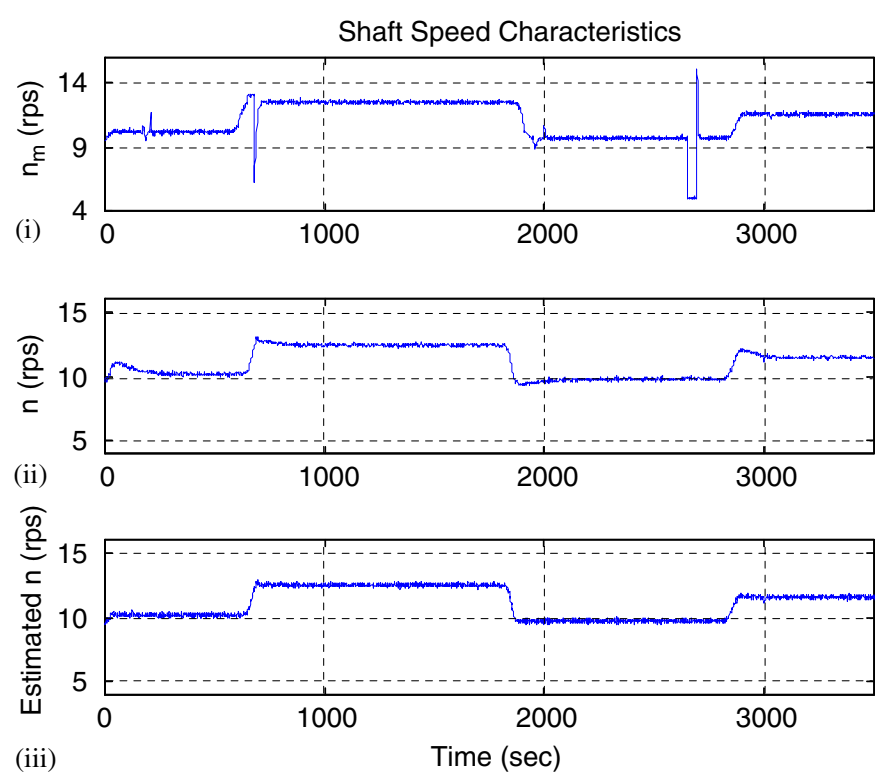

Fig. 4. Shaft speed (i) measurement from the original benchmark, (ii) true state from the fault-tolerant system, (iii) estimate that is fed back in the fault-tolerant system.
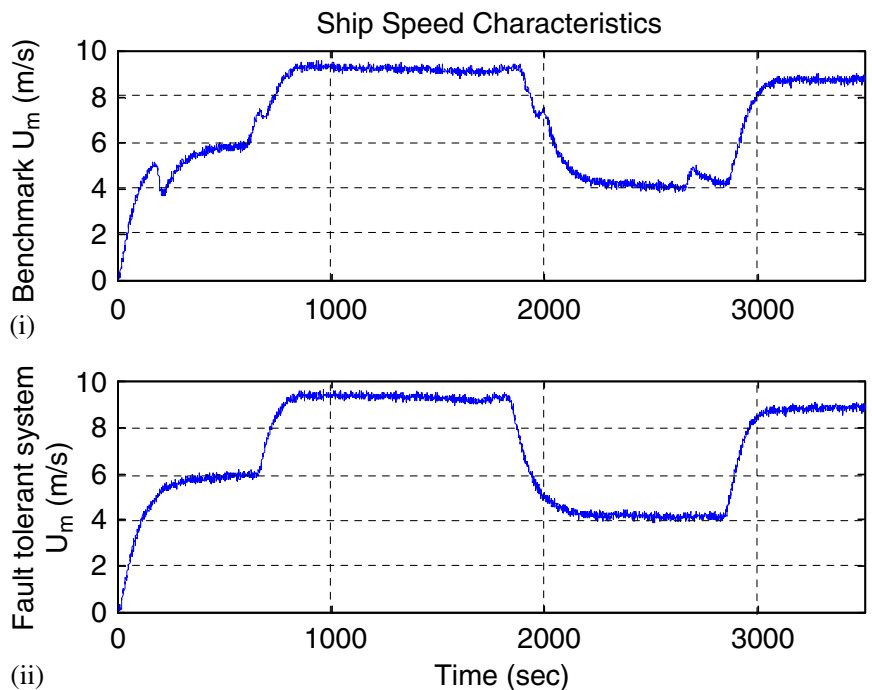

Fig. 5. Ship speed (i) measurement from the original benchmark, (ii) measurement from the fault-tolerant system.

Table 3

Benchmark fault event profile

\begin{tabular}{lll}
\hline Fault type & Approx. magnitude & Sustaining duration \\
\hline$\Delta \theta$ & 0.4 & $180 \mathrm{~s}-210 \mathrm{~s}$ \\
$\Delta n$ & 0.5 & $680 \mathrm{~s}-710 \mathrm{~s}$ \\
$\Delta \dot{\theta}_{i n c}$ & $10^{-5}\left(s+10^{-5}\right)^{-1}$ & $800 \mathrm{~s}-1700 \mathrm{~s}$ \\
$\Delta \theta$ & -0.35 & $1890 \mathrm{~s}-1920 \mathrm{~s}$ \\
$\Delta n$ & -9.6 & $2640 \mathrm{~s}-2670 \mathrm{~s}$ \\
$\Delta k_{y}$ & $20 \% k_{y}$ & $3000 \mathrm{~s}-3500 \mathrm{~s}$ \\
\hline
\end{tabular}

can be seen that sensor fault masking has been successfully achieved. Note that in the process, no switching action is taken. This is due to the self-adjustable nature of the fault masking estimator. In addition, we are able to retain the controller in the original benchmark despite the presence of an incipient fault and a parametric fault that are not directly measured by any sensor. This robustness is attributed to the less severe nature of the two additional faults, and to the enhanced observability of the modified system configuration.

The contribution of the fault masking estimator can be thought of as an automatic compensation of a sensor fault which is recognizable from one of two sensor measurements by utilizing analytic redundancy, while a third sensor would be required with traditional fail-operational technology.

The reader having an interest in reproducing the numerical results presented in the paper is referred to Izadi-Zamanabadi and Blanke (1999) for the standard set of parameter values of the benchmark. Only the sequence of fault occurrence is given in Table 3 to facilitate the reading of the plots.

The table reflects an important difference in the treatment of sensor faults in this paper from that in all 
other reported ship propulsion benchmark studies. The sensor faults in this paper are modeled as measurement deviations from the fault-free values, rather than absolute deviations from the zero outputs.

\section{Discussion}

Our investigation is continuing in the following three areas.

(i) The difficulty presented by the hard nonlinearities in the benchmark, which an extended Kalman estimator is incapable of handling, is circumvented by staying with a small signal model during analysis and design. The most challenging task of stability analysis of the closed-loop system is left with the simulation. Only a small signal analysis where the estimator is identified as a parametric LTI model has been performed (Thavamani, 2002). A more rigorous nonlinear, closed-loop stability analysis without small signal LTI approximations is being sought.

(ii) The current controller which is inherited from the original benchmark is constructed based on a model without the estimator dynamics in the loop. Although the dynamic effect of the estimator to the loop has been minimized by the use of a forgetting factor in the estimator, in general a controller design that includes the estimator dynamics in the control design model is needed.

(iii) While all fault effect parameters enter explicitly into the estimator design model in order to gain greater accuracy of the virtual measurement estimates, the nonsensor faults $\Delta \dot{\theta}_{i n c}$ and $\Delta k_{y}$ have not been included in the controller design model. This is acceptable because these faults have only minor effects on the system performance. In a more general situation, however, effort to compensate non-sensor faults may be required.

\section{Acknowledgements}

The support of NASA (NCC-1-02009) and NSF (ECS9615956) is gratefully acknowledged by the first two authors. The authors also sincerely thank Dr. IzadiZamanabadi of Aalborg University for his most generous assistance with the ship propulsion benchmark.

\section{References}

Amann, P., Perronne, J.M., Gissinger, G.L., \& Frank, P. M. (1999). Benchmark application of fuzzy observers to fault detection on a ship propulsion system. Proceedings of European control conference, Karlsruhe, Germany, F1062-2.
Blanke, M., Izadi-Zamanabadi, R., \& Lootsma, T. F. (1998). Fault monitoring and re-configurable control for a ship propulsion plant. Journal of Adaptive Control and Signal Processing, 12(8), 671-688.

Blanke, M., Kinnaert, M., Lunze, J., \& Staroswiecki, M. (2003). Diagnosis and fault-tolerant control. Berlin, Germany: Springer.

Blanke, M., \& Lootsma, T. F. (1999). Adaptive observer for diesel fault detection in a ship propulsion benchmark. Proceedings of European control conference, Karlsruhe, Germany, F1062-4.

Bonivento, C., Paoli, A., \& Marconi, L. (2003). Fault-tolerant control for the ship propulsion system. Control Engineering Practice, 11(5), 483-492.

Chow, E. Y., \& Willsky, A. S. (1984). Analytical redundancy and the design of robust detection systems. IEEE Transaction on Automatic Control, 29(7), 603-614.

Edwards, C., \& Spurgeon, S. K. (2000). A sliding mode observer based FDI scheme for the ship benchmark. European Journal of Control, 6(4), 341-356.

Izadi-Zamanabadi, R. (1999). Fault-tolerant supervisory control system analysis and logic design. Ph.D. thesis, Department of Control Engineering, Aalborg University, Denmark.

Izadi-Zamanabadi, R., Amam, P., Blanke, M., Concquemet, V., Gissinger, G.L., Kerrian, E.C., et al. (2000). Ship propulsion control and reconfiguration. In: K. J. Åström, P. Albertos, M. Blanke, A. Isidori, W. Schaufelberger, \& R. Santz (Eds.), Control of complex systems. Berlin, Springer.

Izadi-Zamanabadi, R., \& Blanke, M. (1999). A ship propulsion system as a benchmark for fault tolerant control. Control Engineering Practice, $7(2), 227-239$.

Izadi-Zamanabadi, R., Blanke, M., \& Katebi, S. (2003). Cheap diagnosis using structural modelling and fuzzy-logic based detection. Control Engineering Practice, 11(4), 415-421.

Kerrigan, E. C., \& Maciejowski, J. M. (1999). Fault tolerant control of a ship propulsion system using model predictive control. Proceedings of European control conference, Karlsruhe, Germany, F1062-3.

Lootsma, T. F. (2001). Observer-based fault detection and isolation for nonlinear systems. Ph.D. thesis, Department of Control Engineering, Aalborg University, Denmark.

Moore, B. C. (1981). Principal component analysis in linear systems: controllability, observability, and model reduction. IEEE Transactions on Automatic Control, 26(1), 17-32.

Patton, R. J. (1997). Fault-tolerant control: the 1997 situation. Proceedings of Safeprocess'97, Hull, UK (pp. 1033-1055).

Schreier, G., \& Frank, P. M. (1999). Fault tolerant ship propulsion control: Sensor fault detection using a nonlinear observer. Proceedings of European control conference, Karlsruhe, Germany, F1062-1.

Thavamani, S. (2002). Observer-based sensor fault-tolerant control of a ship propulsion benchmark. Master of Science Thesis, Binghamton University, USA.

Wu, N. E. (2004). Coverage in fault tolerant control. Automatica, 40(4), 537-548.

Wu, N. E., Zhang, Y. M., \& Zhou, K. (2000). Detection, estimation, and accommodation of loss of control effectiveness. International Journal of Adaptive Control and Signal Processing, 14(7), 775-795.

Wu, N. E., Zhou, K., \& Salomon, G. (2000). Reconfigurability in linear time-invariant systems. Automatica, 36(11), 1767-1771.

Zhang, Y. M., \& Wu, N. E. (1999). Fault diagnosis for a ship propulsion benchmark: part I. Proceedings of 14th IFAC world congress, Beijing, PRC (Vol. O, pp. 569-574). 